\title{
Optimal Monopoly Price Paths with Expanding Networks
}

\author{
JEAN GABSZEWICZ \\ CORE - Université Catholique de Louvain \\ FILOMENA GARCIA * \\ UECE - Technical University of Lisbon
}

\begin{abstract}
In this paper, we survey the literature on optimal pricing of a monopolist operating on a network industry. We also describe explicitly the optimal path of prices for a monopolist facing an expanding demand due to the presence of network effects. The optimal path of prices appears as a function of the network effect intensity and horizon length. Prices are increasing over time and for very low network effect intensity, or very high horizon length, the monopolist will offer the good at zero price in the initial period.
\end{abstract}

\section{Introduction}

This paper has two objectives: first to survey briefly the main results in the literature about the pricing decisions of a monopolist who sells a good subject to network externalities and second, to uncover the monopolist's optimal strategy in a discrete time model and study how it depends on the intensity of the preference for the network and on the horizon's length.

Since Rohlfs (1974) first introduced the term "network effect" to indicate that utility may be increasing in the number of consumers of the good, scholars have been interested in the implications of this feature to the economic analysis, investigating issues like the incentives to innovate, the choice of compatibility among competing networks, the emergence of standards and the pricing of goods subject to network externalities.

However, and quite surprisingly, the dynamic properties of the network effects have received little attention and in most cases (take for instance the model by Rohlfs, 1974), the dynamic properties are discussed without actually modelling a time dependent framework, but by considering that equilibrium is perfect foresight. Likewise, the pricing decisions under the hypothesis of expanding demand have also received little attention.

\footnotetext{
* Mailing address: Research Unit on Complexity and Economics - Technical University of Lisbon, Rua Miguel Lúpi, 20, 1249-178 Lisboa, Portugal. E-mail: fgarcia@iseg.ut.pt. We thank the editor of RNE for his suggestions and examples. All errors are our responsibility.
} 
Several models have been considered to comply with consumption externalities. In the present paper, we will discuss the network effects that are generated from past consumption of the good. As in Katz and Shapiro (1985, 1994), and Farrell and Saloner (1985), the network takes one period of time to be constituted so that consumers derive utility in each period from the volume of past sales. This type of network externalities can be found when the quality of the good depends on some "word of mouth" or "learning by doing" process, according to the terminology in Bensaid and Lesne (1996). Early consumers allow firms to improve their product or to offer better services and assistance in later periods, which increases the benefit of consuming the good for later users. Similar properties are found in the so-called reputation goods (see Rogerson, 1982), or goods which require a learning period. For instance, consider the market for a reputation good when reputation increases with the size of its market. The initial buyers of such a good spread information about how to use it and enjoy its consumption. In addition, for many goods, the size of the initial network of buyers determines the availability of complementary services the seller would be willing to develop around the variant that is initially supplied. Examples are: a bank and the number of branches which increases with the number of its customers, a new machine and the number of its components when they are available to subsequent buyers, like in the case of a computer whose number of compatible programs increases with the number of its initial buyers. Notice however, that such product improvements or reputation effects need some period of time to be finalized, creating a time lag between the creation of the network and its effect on the utility of subsequent buyers. The interdependence between demand and past consumers' decisions implies that the monopolist's pricing decision must consist of an intertemporal strategy: the price chosen in early periods influences future sales and profits.

Dhebar and Oren (1985, 1986) have built upon Rohlf's framework to obtain the optimal path of prices for a monopolist both in the case of linear and nonlinear prices. By doing so, they are able to draw the conclusion that price-cost margins are increasing through time. Dhebar and Oren $(1985,1986)$ include also the possibility that consumers may not be completely certain about their peers ordering in the economy and as such need to anticipate the future network growth. Then they perform comparative statics on the degree of optimism of consumers regarding the growth of the network. Their conclusion is that when consumers expect a high network growth, the monopolist will set a high price. However, Dhebar and Oren use models whose complexity does not allow identifying explicitly the optimal path of prices (their results rely on numerical methods).

In this paper we describe explicitly the monopolist's optimal strategy and we examine how it depends on the intensity of the preference for the network and on the horizon's length. The formal proofs of the results described here can be found in Gabszewicz and Garcia (2007). In initial periods, the network effect is not the main driver of consumption, thus the monopolist has no incentive to set the monopoly price. On the contrary, setting a lower price attracts consumers and increases the demand in future periods. The incentive to set low prices is, nevertheless, decreasing in time because, as the horizon becomes closer, the weaker the interest of increasing further future demands. As a consequence, we first observe that the path of optimal prices is increasing through time and that this price is steeper when the network effect intensity is weaker. We also observe that this path of prices approaches the sequence of instantaneous monopoly prices corresponding to the sequence of accumulated demands, while being exactly equal to this monopoly price in the last period. Furthermore, for large values of the network effect intensity, we show that the 
monopolist chooses initial prices to be zero, indicating that he would even set a negative price if this would be admissible.

Firm's usage of the "penetrating price" strategy can be often observed. Software is usually offered at very low price (or even given for free) in initial periods, whereas updates are expensive (Adobe Acrobat, SpamNet); similarly, phone companies or network providers have low price deals for new services (See Ackere and Reyniers, 1995). ${ }^{1}$ Another example would be pricing for Internet services: the service can be initially free but charging is introduced later on, once a network is constituted. Examples are the sites for SMS services or dating sites. The same trend can be observed in online newspaper publishing as providers start to charge a subscription to use the service that once was offered for free.

The penetrating price result can also be found in Cabral et al (1999). They study monopoly pricing in a network industry and conclude that introductory pricing occurs if consumers are price takers, if there is incomplete information about demand or asymmetric information about cost. We show that introductory pricing occurs in a setup where information is complete and symmetric.

The penetrating price strategy has to be contrasted with the intertemporal pricing of durable goods which are initially priced very high, and then reduced progressively, reflecting the monopoly interest of discriminating among consumers with different reservation prices (Coase 1972).

A different approach to dynamic monopoly pricing within a continuous time framework can be found in Clarke et al (1982). These authors analyse the problem of optimal intertemporal pricing for a monopolist when current and past output affect future cost and/or demand conditions through experience in production and/or consumption. The paper shows that learning by doing implies a decreasing path of prices through time if learning leads to a decrease in marginal cost. Also, consumer's experience effects imply non-monotonicity of the price paths because increased consumption leads beyond some threshold value to market saturation. Despite their general approach, Clarke et al. (1982) do not discuss network effects in demand.

We provide in the following sections a general presentation of the results obtained in Gabszewicz and Garcia (2007).

\section{The model and results}

Consider a monopolist operating in a market for a finite number of periods and selling a durable good at constant average cost set to zero.

At each period $t, t=1, \ldots, T$, a new cohort of consumers enters the market. Consumers value the good for its intrinsic utility and for the network benefits that it entails. The more consumers buy the good the higher is its value for each individual. Consumers are differentiated according to their stand-alone value. They are distributed uniformly in $[0,1]$ by increasing order of intrinsic valuation for the good.

The demand for the good at time $t$, is denoted by $D_{t}\left(p_{t}, p_{t-1}, \ldots, p_{0}\right)$. The utility of agent $v$ at time $t$ is given by:

\footnotetext{
${ }^{1}$ Vodafone just released an email service on the mobile phone which is free for a certain period but will be priced positively in the future.
} 


$$
u_{t}\left(v ; p_{t-1}, \ldots, p_{1}\right)=v+\alpha \sum_{j=1}^{t} D_{t-j}\left(p_{t-j}, \ldots, p_{1}\right)
$$

where parameter $\alpha, \alpha>0$, measures the intensity of the network effect.

First, we derive the demand function for the product in each period $t, t=1, \ldots, T$. Since the consumer, in her decision problem at period $t$, only considers the network constituted in the past periods, the functional form of demand in each period is independent of the subsequent periods and depends only on prices $p_{\tau}, \tau=1, \ldots t$. Let us begin with $T=2$. The demand at period 1 is given by:

$$
D_{1}\left(p_{1}\right)=1-p_{1}, \quad 1 \geq p_{1} \geq 0
$$

In period 2, taking into account the network effect created in period 1 , we identify the consumer $v$ that is indifferent between buying the product and not buying in period 2 by the condition: $v+\alpha\left(1-p_{1}\right)-p_{2}=0$, namely, $\hat{v}=p_{2}-\alpha\left(1-p_{1}\right)$. Thus, demand in period 2 is given by: $D_{2}\left(p_{2}, p_{1}\right)=1-p_{2}+\alpha\left(1-p_{1}\right)$. Proceeding by induction, it is easy to see that the demand in period $t \leq T$ obtains as:

$$
D_{t}\left(p_{t}, \ldots, p_{1}\right)=(1+\alpha)^{t-1}-\alpha \sum_{j=2}^{t}(1+\alpha)^{t-j} p_{j-1}-p_{t}
$$

Notice that when $\alpha>1$, the demand may react with more intensity to previous prices than to the current price. This is so because under this condition, lower initial prices increase the value of the good for subsequent users. On the contrary, for $\alpha<1$, the effect of initially low prices tends to vanish with time. An alternative expression for the demand obtains as

$$
D_{t}\left(p_{t}, \ldots, p_{1}\right)=1-p_{t}+\alpha \sum_{j=1}^{t} D_{t-j}
$$

The demand in period $t$ is a linear decreasing function of $p_{t}$ with the constant term increasing in $t$. All the above expressions for demands at period $t$ must satisfy the fact that demand can never exceed 1.

The total profit function with horizon $T$ is written as:

$$
\Pi\left(T, p_{T}, \ldots, p_{1}\right)=\sum_{k=1}^{T} p_{k} D_{k}=\sum_{k=1}^{T} p_{k}\left((1+\alpha)^{k-1}-\alpha \sum_{j=2}^{k}(1+\alpha)^{k-j} p_{j-1}-p_{k}\right)
$$

\section{The optimal price policy}

Consider the following three conditions: 


$$
\begin{aligned}
& \alpha<\frac{1}{T-1}, \\
& \frac{1}{T-1} \leq \alpha \leq \frac{2}{T-1}, \\
& \alpha>\frac{2}{T-1} .
\end{aligned}
$$

Proposition 1: Under C.1, the set of values given by:

$$
p_{t}(T)=\frac{1-(T-t) \alpha}{2-(T-1) \alpha}, t=1, \ldots T
$$

is the unique solution to the monopolist's profit maximization problem.

Proof: See Gabszewicz and Garcia (2007).

The condition C. 1 is sufficient to guarantee that the profit function of the monopolist is concave. It is then easy to obtain (3) as the vector of prices, which maximizes the profit.

It is interesting to discuss briefly how this solution depends on the main ingredients of the model, namely on $t$, on the time horizon, $T$, and the network effect intensity parameter $\alpha$. The price quoted by the monopolist in period $t$ is decreasing on $T$. That is, a monopolist who will operate for a longer period of time has an incentive to set a lower price per period, as the gains in terms of future demand more than compensate for the loss in present profit. In addition, we obtain that prices increase over time. This effect is stronger, the higher the intensity of the network effect (the cross derivative of (3) w.r.t. $t$ and $\alpha$ is positive). The economic implications are clear: the monopolist is more willing to use the increasing pattern of prices if the industry in which he operates has strong network effects.

We analyse now the equilibrium demands, $D t, t=1, \ldots, T$, computed at optimal prices $p_{t}(T), \ldots, p_{1}(T)$, namely

$$
D_{t}\left(p_{t}(T), \ldots, p_{1}(T)\right)=\frac{1}{2-\alpha(T-1)}
$$

First notice that the value of each demand at period $t$ evaluated at optimal prices is smaller than 1, which means that the monopolist does not cover the market completely. Furthermore, we find that this value is constant, given the horizon $T$. Finally, it is increasing with the horizon $T$ and decreasing with $\alpha$. The period $t$ profits at the optimal solution $\pi_{t}(T)$ obtain as:

$$
\pi_{t}(T)=p_{t}(T) D_{t}\left(p_{t}(T), \ldots, p_{1}(T)\right)=\frac{1-(T-t) \alpha}{(2-\alpha(T-1))^{2}}
$$

Therefore, the per-period profits $\pi_{t}(T)$ are increasing over time and also increasing with the strength of the network effect $\alpha$ (under C.1). Total profits evaluated at optimal prices, given the horizon $T$, obtain as 


$$
\pi\left(T, p_{t}(T), \ldots, p_{1}(T)\right)=\sum_{t=1}^{T} \pi_{t}(T)=\frac{T}{2(2-(T-1) \alpha)} .
$$

An immediate conclusion is that total profits are an increasing function of the time horizon, as well as of the network effect intensity parameter $\alpha$. The more sensitive are the agents to the network, the higher is the surplus that the monopolist is able to extract through the appropriate pricing strategy.

Now assume that C.2 or C.3 holds. In the first case, total profits are still strictly concave but some, or all, components of the vector

$$
\left\{p_{t}(T)=\frac{1-(T-t) \alpha}{2-(T-1) \alpha}\right\}_{t=1}^{T}
$$

must be equal to 0 since we constrain all prices to be non-negative. When $\alpha$ increases beyond the value $1 /(T-1)$, the strategy given by (3) yields $p_{1}(T)<0$, which we rule out as an equilibrium in this model. In the second case, the total profit function is no longer concave, but convex. Accordingly, the solution to the monopolist's problem occurs at the boundary of the admissible domain of prices, namely $\left\{\left(p_{1}, \ldots, p_{T}\right): 0 \leq D_{t}\left(p_{t}, \ldots, p_{1}\right) \leq 1, t=1, \ldots, T\right\}$. The next proposition characterizes the optimal path of prices for under condition C.2 or C.3.

Proposition 2: Under C.2 or C.3, the set of values given by

$$
\begin{aligned}
& p_{1}(T)=0 \\
& p_{t}(T)=\alpha(t-1), t=2, \ldots, T
\end{aligned}
$$

is the unique solution to the monopolist's profit maximization problem.

Proof: See Gabszewicz and Garcia (2007).

When the network effect intensity parameter and the horizon length meet condition C.1, the market is never saturated: given the weakness of the network effect intensity, the horizon length is not sufficiently remote for the monopolist to cover the market and keep it covered for the remaining periods. When conditions C.2 and C.3 are fulfilled, namely, when the network effect intensity is sufficiently strong, the monopolist is induced to saturate the market from the very beginning and keep it covered for all subsequent periods. Notice that when the horizon length tends to infinity, the domain of the network effect intensity values to which there corresponds an interior solution (that is, in which zero pricing is not practised), tends to a set of measure zero. Thus, we conclude that when the horizon is sufficiently remote, zero pricing in the starting period should be the rule. 


\section{Conclusion}

In this paper, we first provided a brief survey of the literature about optimal pricing of a monopolist operating in a network industry. Then we considered a model depicting a monopoly market with delayed network externalities and identified the intertemporal optimal price path for the monopolist. For this model, our analysis was developed along the values of two major parameters, namely, the network effect intensity and the horizon length, which are related to each other by conditions C.1 to C.3.

In concluding the paper, we consider some further questions to be investigated in future research. In our framework, consumers arrive in the market by successive waves and cannot delay consumption, which implies that they cannot arbitrage between periods when making their purchase decisions. An alternative assumption would be to suppose that consumers might postpone their consumption. In this case, they should anticipate future prices and choose their instantaneous consumption according to their expectations. This constitutes a completely different version of the problem, which would certainly be of interest. It would also be natural to consider the same problem under more complex market structures, like oligopoly, with firms behaving strategically. Then, strategies would be defined as price paths and we would be looking for a Nash equilibrium defined with respect to these strategies.

Finally, this paper can also serve the purpose of analysing the incentives of a monopolist to set negative prices, or in other words, to offer initial buyers a compensation for the usage of the good. This widespread practise can be justified by the presence of network externalities in consumption. Negative prices could be observed in our framework if such strategies were available to the monopolist.

\section{$5 \quad$ References}

Ackere, A. and D. Reyniers (1995) "Trade-ins and Introductory Offers in a Monopoly," RAND Journal of Economics, 26: 58-74.

Bensaid, B. and J.-P. Lesne (1996) "Dynamic Monopoly Pricing with Network Externalities,” International Journal of Industrial Organization, 14: 837-855.

Cabral, L., D. Salant and G. Woroch (1999) "Monopoly Pricing with Network Externalities”, International Journal of Industrial Organization, 17: 199-214.

Clarke, F. and Darrough, M. and J. Heineke (1982) "Optimal Pricing Policy in the Presence of Experience Effects,” The Journal of Business, 55: 517-530.

Coase, R. (1972) “Durability and monopoly”, Journal of Law and Economics, 15: 143149.

Dhebar, A. and S. Oren (1985) "Dynamic Nonlinear Pricing in Networks with Interdependent Demand,” Operations Research, 34: 384-394. 
Dhebar, A. and S. Oren (1985) "Optimal Dynamic Pricing for Expanding Networks," Marketing Science, 4: 336-351.

Farrell, J. and G. Saloner (1985) “Standardization, Compatibility, and Innovation,” RAND Journal of Economics, 16: 70-83.

Gabszewicz, J. and F. Garcia (2007) "A Note on Expanding Networks and Monopoly Pricing,” forthcoming, Economics Letters.

Katz, M. and C. Shapiro (1985) "Network Effect, Competition, and Compatibility," American Economic Review, 75: 424-440.

Katz, M. and C. Shapiro (1994) “System Competition and Network Effects," Journal of Economic Perspectives, 8: 93-115.

Rogerson, W. (1983) "Reputation and Product Quality,” The Bell Journal of Economics, 14: 508-516.

Rohlfs, J. (1974) “A Theory of Interdependent Demand for a Communication Service,” Bell Journal of Economics, 5: 16-37. 